\title{
Summing Up All Genus Free Energy of ABJM Matrix Model
}

\author{
Hiroyuki Fuji ${ }^{a *}$, Shinji Hirano ${ }^{b \dagger}$, and Sanefumi Moriyama ${ }^{c \ddagger}$ \\ ${ }^{a, b}$ Department of Physics \\ Nagoya University \\ Nagoya 464-8602, Japan \\ ${ }^{c}$ Kobayashi Maskawa Institute \\ Nagoya University \\ Nagoya 464-8602, Japan
}

\begin{abstract}
The localization technique allows us to compute the free energy of the $U(N)_{k} \times$ $U(N)_{-k}$ Chern-Simons-matter theory dual to type IIA strings on $A d S_{4} \times C P^{3}$ from weak to strong 't Hooft coupling $\lambda=N / k$ at finite $N$, as demonstrated by Drukker, Mariño, and Putrov. In this note we study further the free energy at large 't Hooft coupling with the aim of testing AdS/CFT at the quantum gravity level and, in particular, sum up all the $1 / N$ corrections, apart from the worldsheet instanton contributions. The all genus partition function takes a remarkably simple form - the Airy function, $\operatorname{Ai}\left(\left(\pi k^{2} / \sqrt{2}\right)^{2 / 3} \lambda_{\text {ren }}\right)$, with the renormalized 't Hooft coupling $\lambda_{\text {ren }}$.
\end{abstract}

*fuji@th.phys.nagoya-u.ac.jp

†hirano@eken.phys.nagoya-u.ac.jp

${ }_{\ddagger}^{\ddagger}$ moriyama@math.nagoya-u.ac.jp 


\section{Introduction and main results}

There has been considerable progress in testing the AdS/CFT conjecture [1] in the large $N$ limit. Through its connection to the spin chain systems, the large $N$ integrability allowed us to successfully check the duality from weak to strong 't Hooft coupling with remarkably high accuracy [2]. In the meantime, there is hardly any test of the AdS/CFT duality at finite $N$. On the one hand, the $1 / N$ corrections are quantum gravity loop corrections and thus very hard to compute on the gravity side. On the other hand, there are indications from the field theory analysis that the integrability breaks down at the non-planar level [3]. So there was virtually no technique to evaluate the $1 / N$ corrections at strong 't Hooft coupling.

However, there has been important progress in the localization technique in supersymmetric gauge theories [4], elucidating ealier works [5, 6]. This provides a powerful method to compute the $1 / N$ corrections at strong 't Hooft coupling in dual CFT's. In [7] the localization technique was applied to $\mathcal{N}=6 U(N)_{k} \times U(N)_{-k}$ Chern-Simons-matter theory, also known as the ABJM theory, dual to type IIA strings on $A d S_{4} \times C P^{3}$ or M-theory on $A d S_{4} \times S^{7} / Z_{k}$ [8]. The partition function of the ABJM theory reduces to the eigenvalue integrals and this defines the ABJM matrix model. Subsequently, the free energy of the ABJM matrix model was computed exactly in the large $N$ limit and order by order in the $1 / N$ expansion [9, 10, 11]. In particular, the genus zero free energy at strong 't Hooft coupling precisely agrees with the tree level SUGRA action. This provided a remarkable check of the AdS/CFT duality and showed the power of the localization technique 1

In this note we study further the free energy of the ABJM theory at strong 't Hooft coupling at finite $N$ with the aim of testing AdS/CFT at the quantum gravity level and, in particular, resum all the $1 / N$ corrections. Our main result is that, apart from the worldsheet instanton contributions, the all genus free energy of the ABJM matrix model sums up to

$$
F^{\mathrm{ABJM}}=\log \left[2 \pi C_{1} \mathrm{Ai}\left(\left[\frac{\pi}{\sqrt{2}}\left(\frac{N}{\lambda}\right)^{2} \lambda_{\text {ren }}^{3 / 2}\right]^{2 / 3}\right)\right],
$$

where $C_{1}=(2 \pi / k)^{-1 / 3} / \sqrt{2}$ and $\lambda=N / k$ is the 't Hooft coupling of $\mathcal{N}=6 U(N)_{k} \times U(N)_{-k}$ Chern-Simons-matter theory, and

$$
\lambda_{\text {ren }}=\lambda-\frac{1}{24}-\frac{\lambda^{2}}{3 N^{2}} .
$$

This non-planar shift/renormalization of the 't Hooft coupling (1.2) partially resums the all genus free energy [11]. As we will see, once this partial resummation is done, the remaining higher genus free energies obey a very simple recursion relation [16] which one can easily solve to find the main result (1.1).

\footnotetext{
${ }^{1}$ See related interesting developments; generalizations to quiver gauge theories [12, the $F$-maximization conjecture [13], computations of superconformal index [14], and tests of three-dimensional dualities [15].
} 
It is worthwhile to note that the shift (1.2) is closely related to the renormalization of the AdS radius (in the $\alpha^{\prime}=1$ unit) [17]:

$$
R_{A d S}^{2}=2^{5 / 2} \pi \sqrt{\lambda-\frac{1}{24}+\frac{\lambda^{2}}{24 N^{2}}} .
$$

In the planar limit $N \rightarrow \infty$, the matrix model shift (1.2) agrees with that of the dual string theory (1.3), as noted in [9, 10] $]^{2}$ However, there appears to be a discrepancy at the nonplanar level; The matrix model suggests a further shift $-3 \lambda^{2} / 8 N^{2}$ to the string/M theory prediction. This may provide a simplest test of AdS/CFT at the quantum gravity level, and it is very important to understand whether and how this discrepancy can be reconciled. We will return to this point later in the discussions.

The organization of the rest of our paper is as follows: In section 2 we briefly review the localization technique and the ABJM matrix model and summarize the result of [9] relevant to our study. In section 3 we give a brief review of the technique to compute the higher genus free energies by using the so-called holomorphic anomaly equation and carry out a partial resummation of the all genus free energy. In section 4 we propose the all genus free energy up to the worldsheet instanton corrections and give a proof of our proposal. In section 5 we summarize our results and discuss the SUGRA one-loop corrections to the free energy. In the appendices we give the technical details of our computations.

\section{The ABJM matrix model}

The localization technique [4] allows us to compute the free energy of the ABJM theory from weak to strong 't Hooft coupling $\lambda=N / k$ at finite $N$. The localization of this theory on $S^{3}$ was carried out by Kapustin, Willet, and Yaakov in [7]; The partition function reduces to the finite dimensional integral over the eigenvalues of two $U(N)$ matrices. This defines the ABJM matrix model. As demonstrated by Drukker, Mariño, and Putrov [9, 11, 10], the free energy can be calculated for arbitrary 't Hooft coupling $\lambda$ and order by order in the $1 / N$ expansion.

The field content of the ABJM theory consists of two $3 \mathrm{~d} \mathcal{N}=2 U(N)$ vector multiplets, $\left(A_{\mu}^{A}, \sigma^{A}, \lambda^{A}, \bar{\lambda}^{A}, D^{A}\right)_{A=1,2}$, which are the dimensional reduction of $4 \mathrm{~d} \mathcal{N}=1$ vector multiplets and two bifundamental chiral multiplets, $\left(\phi^{I}, \bar{\phi}^{I}, \psi^{I}, \bar{\psi}^{I}, F^{I}, \bar{F}^{I}\right)_{I=1,2}$, in the representation $(N, \bar{N})$, and their duals in $(\bar{N}, N) \cdot 3$ This theory enjoys the nilpotent Grassmann-odd symmetry generated by $\bar{\delta}=\bar{\epsilon} \bar{Q}$ where $\bar{\epsilon}$ being the two-component complex Killing spinor on $S^{3}$ with the normalization $\bar{\epsilon} \epsilon=1$ and $\bar{Q}$ is the $\mathcal{N}=1$ supercharge.

\footnotetext{
${ }^{2}$ In 9 , the unequal rank $U\left(N_{1}\right) \times U\left(N_{2}\right)$ case [18] was also studied, and the generalization of (1.2) precisely agrees with the SUGRA prediction [19] in the large $N$ limit.

${ }^{3}$ In Euclidean space, the barred fields are not complex conjugate of the unbarred fields but independent of them.
} 
The partition function is invariant under the deformation of the action by any $\bar{\delta}$-exact terms, since the $\mathcal{N}=6 U(N)_{k} \times U(N)_{-k}$ Chern-Simons-matter action and the vacua are invariant under the $\bar{\delta}$-transformation. This has an important ramification: By deforming the action by $t \bar{\delta} V$ with a positive definite $\bar{\delta} V$, as we send $t$ to infinity, the path integral localizes on the saddle point of $\bar{\delta} V$. A particularly convenient choice of $\bar{\delta} V=\bar{\delta} V_{\text {gauge }}+\bar{\delta} V_{\text {matter }}$ is [7, 20]

$$
\begin{aligned}
\bar{\delta} V_{\text {gauge }} & =\bar{\delta} \delta \operatorname{Tr}_{U(N)}\left(\frac{1}{2} \bar{\lambda}^{A} \lambda^{A}-2 D^{A} \sigma^{A}\right)=S_{\mathrm{YM}}, \\
\bar{\delta} V_{\text {matter }} & =\bar{\delta} \delta\left(\bar{\psi}^{I} \psi^{I}-2 i \bar{\phi}^{I}\left(\sigma^{1}-\sigma^{2}\right) \phi^{I}-\bar{\phi}^{I} \phi^{I}\right)=S_{\mathrm{m}},
\end{aligned}
$$

where $S_{\text {YM }}$ and $S_{\mathrm{m}}$ are the $\mathcal{N}=2$ super Yang-Mills action and the bifundamental matter action on the unit sphere, respectively. Their saddle points are given by [7]

$$
A_{\mu}^{A}=\phi^{I}=0, \quad D^{A}=-\sigma^{A}=\text { const } .
$$

The localization action $S_{\mathrm{YM}}+S_{\mathrm{m}}$ vanishes on the saddle points. Thus, in the $t \rightarrow \infty$ limit, the only contribution comes from (1) the classical Chern-Simons-matter action $S_{c l}$ evaluated on the saddle points and (2) the quadratic fluctuations about the saddle points in the localization action. The latter gives the one-loop determinants, and thus schematically the partition function becomes

$$
Z^{\mathrm{ABJM}}=\int d \sigma^{1} d \sigma^{2} \frac{\operatorname{det} \Delta_{F}\left(\sigma^{A}\right)}{\operatorname{det} \Delta_{B}\left(\sigma^{A}\right)} \exp \left(-S_{\mathrm{cl}}\left(\sigma^{A}\right)\right),
$$

where $\Delta_{B}$ and $\Delta_{F}$ denote the Laplacians on $S^{3}$ for the bosonic and fermionic fluctuations, respectively.

Diagonalizing the $U(N)$ matrices $\sigma^{i=1,2}$ and integrating their angular parts yields the Vandermonde determinants which are cancelled by the factors from the one-loop determinants. The net result is given by [7, 22, 9, 10]

$$
\begin{gathered}
Z^{\mathrm{ABJM}}=\frac{1}{(N !)^{2}} \int \prod_{i=1}^{N} \frac{d \mu_{i}}{2 \pi} \prod_{a=1}^{N} \frac{d \nu_{a}}{2 \pi} \frac{\prod_{i<j}\left(2 \sinh \left(\frac{\mu_{i}-\mu_{j}}{2}\right)\right)^{2} \prod_{a<b}\left(2 \sinh \left(\frac{\nu_{a}-\nu_{b}}{2}\right)\right)^{2}}{\prod_{i, a}\left(2 \cosh \left(\frac{\mu_{i}-\nu_{a}}{2}\right)\right)^{2}} \\
\times e^{-\frac{1}{2 g_{s}}\left(\sum_{i} \mu_{i}^{2}-\sum_{a} \nu_{a}^{2}\right)},
\end{gathered}
$$

where $g_{s}=2 \pi i / k . \mu_{i}$ 's and $\nu_{a}$ 's are the eigenvalues of the two $U(N)$ matrices $\sigma_{i}$, and the hyperbolic functions are the one-loop determinant contributions. Those in the numerator are from the vector multiplets, whereas those in the denominator are from the bifundamental matter multiplets which give the coupling between the two $U(N)$ factors. The eigenvalue integral (2.5) defines the ABJM matrix model.

The planar limit of the ABJM matrix model can be solved by the standard technique [9, 11, 10]. In the large $N$ limit, the partition function (2.5) is dominated by the saddle 
points:

$$
\begin{aligned}
& \mu_{i}=\frac{t_{1}}{N_{1}} \sum_{j \neq i}^{N_{1}} \operatorname{coth} \frac{\mu_{i}-\mu_{j}}{2}+\frac{t_{2}}{N_{2}} \sum_{a=1}^{N_{2}} \tanh \frac{\mu_{i}-\nu_{a}}{2}, \\
& \nu_{a}=\frac{t_{2}}{N_{2}} \sum_{b \neq a}^{N_{2}} \operatorname{coth} \frac{\nu_{a}-\nu_{b}}{2}+\frac{t_{1}}{N_{1}} \sum_{i=1}^{N_{1}} \tanh \frac{\nu_{a}-\mu_{i}}{2},
\end{aligned}
$$

where $t_{i}=g_{s} N_{i}$ and $N_{1}=-N_{2}=N$. In solving these equations, one first considers the problem for positive $N_{1}$ and $N_{2}$ and then analytically continues the result to the negative $N_{2}=-N_{1}=-N$.

The effective potential which leads to the above saddle point equations consists of attractive harmonic potentials and logarithmic Coulomb repulsions for the eigenvalues $\mu_{i}$ 's and $\nu_{a}+i \pi$ 's. Since the Coulomb repulsions have the strength $t_{i}$ 's, when the 't Hooft couplings $t_{i}$ 's are zero, the harmonic potentials dominate and the eigenvalues collapses to zero. As we increase $t_{i}$ 's, the Coulomb repulsions kick in and spread the eigenvalues along two line intervals; The $\mu_{i}$ 's condense in $\mathcal{C}_{1}$ on the real axis, and the $\nu_{a}+i \pi$ 's in $\mathcal{C}_{2}$, the interval separated from $\mathcal{C}_{1}$ by $+i \pi$. Thus the ABJM matrix model is a two-cut model.

Similarly to the case of the standard matrix models, one defines the resolvent by

$$
\omega(z)=g_{s}\left\langle\sum_{i=1}^{N_{1}} \operatorname{coth} \frac{z-\mu_{i}}{2}\right\rangle+g_{s}\left\langle\sum_{a=1}^{N_{2}} \tanh \frac{z-\nu_{a}}{2}\right\rangle .
$$

To make more direct contact with the standard matrix models, it is useful to introduce the new variable $Z=e^{z}$. Then the resolvent is expressed as

$$
\omega(z) d z=-t \frac{d Z}{Z}+2 g_{s}\left\langle\sum_{i=1}^{N_{1}} \frac{d Z}{Z-e^{\mu_{i}}}\right\rangle+2 g_{s}\left\langle\sum_{a=1}^{N_{2}} \frac{d Z}{Z+e^{\nu_{a}}}\right\rangle,
$$

where $t=t_{1}+t_{2}$. As studied in [21], the saddle point equations (2.6) and (2.7) imply that the discontinuity of the resolvent $\omega_{0}(z) \equiv \lim _{N \rightarrow \infty} \omega(z)$ at large $N$ is given by

$$
\begin{aligned}
& z=\frac{1}{2}\left(\omega_{0}(z+i \epsilon)+\omega_{0}(z-i \epsilon)\right) \quad \text { on } \mathcal{C}_{1}, \\
& z=\frac{1}{2}\left(\omega_{0}(z+i \pi+i \epsilon)+\omega_{0}(z+i \pi-i \epsilon)\right) \text { on } \mathcal{C}_{2} .
\end{aligned}
$$

It then follows that the function

$$
f(Z)=e^{t}\left(e^{\omega_{0}}+Z^{2} e^{-\omega_{0}}\right)
$$

is regular everywhere on the complex plane. This has the asymptotic behavior $f(Z) \stackrel{Z \rightarrow \infty}{\longrightarrow} Z^{2}$ and $f(Z) \stackrel{Z \rightarrow 0}{\longrightarrow} 1$. With these boundary conditions, the function $f(Z)$ is uniquely determined:

$$
f(Z)=Z^{2}-\zeta Z+1
$$


Meanwhile, from (2.12) one has

$$
\omega_{0}(Z)=\log \left[\frac{e^{-t}}{2}\left(f(Z)-\sqrt{f(Z)^{2}-4 e^{2 t} Z^{2}}\right)\right] .
$$

Parameterizing the branch cuts $\mathcal{C}_{1}$ and $\mathcal{C}_{2}$ on the $Z$-plane by $[1 / a, a]$ and $[-b,-1 / b]$, respectively, the inside of the square root can be written as the polynomical $(Z-a)(Z-1 / a)(Z+$ $b)(Z+1 / b)$, and one can then identify

$$
\zeta=\frac{1}{2}\left(a+\frac{1}{a}-b-\frac{1}{b}\right), \quad e^{t}=\frac{1}{4}\left(a+\frac{1}{a}+b+\frac{1}{b}\right) .
$$

Once the resolvent is found, the 't Hooft couplings $t_{i}$ can be computed from

$$
t_{i}=\frac{1}{4 \pi i} \oint_{A_{i}} \omega_{0}(z) d z,
$$

and the genus zero free energy from

$$
\frac{\partial F_{0}}{\partial s}=\pi i t-\frac{1}{2} \oint_{B} \omega_{0}(z) d z
$$

where $s=\frac{1}{2}\left(t_{1}-t_{2}\right)$. The contour $A_{i}$ encircles the branch cut $\mathcal{C}_{i}$, whereas the contour $B$ is the cycle dual to the $A_{i}$ cycles. The first term in the RHS of (2.17) is due to the pole at $Z=0$ in (2.9). These contour integrals (2.16) and (2.17) are hard to carry out. However, once the derivative is taken w.r.t. $\zeta$, it becomes easy to perform the integration.

After the analytic continuation $t_{2} \rightarrow-t_{1}=-2 \pi i \frac{N}{k}=2 \pi i \lambda$, the answer for the ABJM matrix model turns out to be [9, 11, 10]

$$
\begin{aligned}
\lambda(\kappa) & =\frac{\kappa}{8 \pi}{ }_{3} F_{2}\left(\frac{1}{2}, \frac{1}{2}, \frac{1}{2} ; 1 ; \frac{3}{2} ;-\frac{\kappa^{2}}{16}\right) \\
\partial_{\lambda} F_{0}(\kappa) & =\frac{\kappa}{4} G_{3,3}^{2,3}\left(\begin{array}{l}
\frac{1}{2}, \frac{1}{2}, \frac{1}{2} \\
0,0,-\frac{1}{2}
\end{array} \mid-\frac{\kappa^{2}}{16}\right)+\frac{\pi^{2} i \kappa}{2}{ }_{3} F_{2}\left(\frac{1}{2}, \frac{1}{2}, \frac{1}{2} ; 1 ; \frac{3}{2} ;-\frac{\kappa^{2}}{16}\right)
\end{aligned}
$$

where $\kappa=-i \zeta$. In particular, at strong coupling $\lambda \gg 1$, these yield

$$
\begin{aligned}
\lambda-\frac{1}{24} & =\frac{\log ^{2} \kappa}{2 \pi^{2}}+\mathcal{O}\left(1 / \kappa^{2}\right), \\
F_{0}(\lambda) & =\frac{4 \sqrt{2} \pi^{2}}{3}\left(\lambda-\frac{1}{24}\right)^{3 / 2}+\mathcal{O}\left(e^{-2 \pi \sqrt{\lambda-\frac{1}{24}}}\right) .
\end{aligned}
$$

Quite remarkably, the first equation shows that, at least in the large $N$ limit, the free energy of the ABJM theory at strong coupling is naturally expressed in terms of the shifted 't Hooft coupling $\lambda-\frac{1}{24}$, rather than $\lambda$, in agreement with the SUGRA prediction [17]. Moreover, the first term in the genus zero free energy precisely agrees with the (minus of) classical 
SUGRA action. As $\lambda=N / k$, this in particular reproduces the tantalizing $N^{3 / 2}$ scaling of the M2-brane theory. Lastly, the exponential corrections to the free energy agree with the expected worldsheet instanton corrections.

These results proclaim the power and relevance of the localization technique to the test of the AdS/CFT conjecture. With this remarkable success, it is natural to ask if the AdS/CFT can be tested beyond large $N$ limit by studying the non-planar corrections in the ABJM matrix model.

\section{$3 \quad$ Higher genus free energies}

The ABJM matrix model is equivalent to the so-called Lens space matrix model [23, 24], which computes the partition function of the $U\left(N_{1}\right) \times U\left(N_{2}\right)$ branch of the Chern-Simons theory on the Lens space $L(2,1)=S^{3} / Z_{2}$, by the analytic continuation $N_{2} \rightarrow-N_{2}$ [22].

Meanwhile, as discussed in [24], the Lens space matrix model arises as the low energy effective theory of D-branes wrapping $S^{3} / Z_{2}$ in the topological A-model on $T^{*}\left(S^{3} / Z_{2}\right)$. At large $N$ the geometric transition takes place and $S^{3} / Z_{2}$ is replaced by the Hirzebruch surface $\mathbb{F}_{0}=\mathbb{P}^{1} \times \mathbb{P}^{1}$, yielding the topological A-model on a non-compact Calabi-Yau space, the canonical line bundle over $\mathbb{F}_{0}$. This is the large $N$ (open/closed string) duality between the Lens space matrix model and the topological $\mathrm{A}$ model on local $\mathbb{F}_{0}$. Furthermore, the A-model on local $\mathbb{F}_{0}$ can be mapped to the B-model on the mirror manifold given by the surface $u v=H(x, y)$, where $H(x, y)=0$ is an elliptic curve and coincides with the spectral curve of the Lens space matrix model. The closed string amplitudes $F^{(g)}(t, \bar{t})$ of the Bmodel, where $t$ is the complex structure moduli, obey the holomorphic anomaly equation of [25] and can be explicitly calculated. In [26] it was conjectured that the holomorphic limit $F^{(g)}(t) \equiv \lim _{\bar{t} \rightarrow \infty} F^{(g)}(t, \bar{t})$ of the amplitudes are given by the genus $g$ free energies of the matrix model whose spectral curve is $H(x, y)=0$. Direct proof was given in [27] built on [28] that the solution of the matrix model loop equation [29] is that of the holomorphic anomaly equation in the $\bar{t} \rightarrow \infty$ limit when the appropriate boundary conditions are imposed. Thus one can use the holomorphic anomaly equation to find the genus $g$ free energies of the ABJM matrix model which is equivalent to the Lens space matrix model by a simple analytic continuation.

\subsection{The holomorphic anomaly equation}

Parameterizing the complex structure moduli by the coordinates $t^{I}$, the holomorphic anomaly equation takes the form [25]: $(g \geq 2)$

$$
\partial_{\bar{I}} F_{g}=\frac{1}{2} C_{\bar{I} \bar{J} \bar{K}} e^{2 K} G^{J \bar{J}} G^{K \bar{K}}\left(D_{J} D_{K} F_{g-1}+\sum_{r=1}^{g-1} D_{J} F_{r} D_{K} F_{g-r}\right)
$$


where $G_{I \bar{J}}=\partial_{I} \partial_{\bar{J}} K$ is the Kähler metric on the moduli space, $C_{I J K}$ is the Yukawa coupling defined by $C_{I J K}=\partial_{I} \partial_{J} \partial_{K} F_{0}$ with $F_{0}$ being the genus 0 free energy, and the covariant derivative $D_{I}$ acts on $F_{g}$ and its derivatives as $\partial_{I}-\Gamma_{I \bullet}^{\bullet}+(2-2 g) \partial_{I} K$. In the local CalabiYau case, the Kähler potential is given by $K=\frac{i}{2}\left(t^{K} \partial_{\bar{K}} \bar{F}_{0}-t^{\bar{K}} \partial_{K} F_{0}\right)$, and thus the moduli space metric is $G_{I \bar{J}}=\operatorname{Im} \tau_{I J}$ with $\tau_{I J} \equiv \partial_{I} \partial_{J} F_{0}[16]$.

When applied to the ABJM matrix model [9], the moduli space becomes one-dimensional and the modulus $t$ is identified with the 't Hooft coupling $\lambda$. Then the Yukawa coupling is $C_{\lambda \lambda \lambda}=4 \partial_{\lambda}^{3} F_{0}(\lambda)=-32 \pi^{3} i \xi$ where

$$
\xi=\frac{2}{\vartheta_{2}(\tau)^{2} \vartheta_{4}(\tau)^{4}}, \quad \tau=\frac{i}{4 \pi^{3}} \partial_{\lambda}^{2} F_{0}(\lambda)+1=i \frac{K^{\prime}\left(\frac{i \kappa}{4}\right)}{K\left(\frac{i \kappa}{4}\right)}
$$

with the normalizations properly adjusted. The genus $g$ free energies are assumed to be of the form

$$
\begin{aligned}
F_{g}(\tau) & =\xi^{2 g-2} f_{g}(\tau) \\
f_{g}(\tau) & =\sum_{k=1}^{3 g-3} \frac{A_{g}^{(k)}(\tau)}{k}\left(\frac{E_{2}(\tau)}{12}\right)^{k}+c_{g}^{0}(\tau),
\end{aligned}
$$

where $A_{g}^{(k)}(\tau)$ 's are the modular forms of weight $6 g-6-2 k$, and $c_{g}^{0}(\tau)$ is the holomorphic ambiguity and a modular form of weight $6 g-6$. The Eisenstein series of weight $2, E_{2}(\tau)$, is a quasi-modular form and can be promoted to the non-holomorphic modular form as

$$
E_{2}(\tau) \longrightarrow \hat{E}_{2}(\tau, \bar{\tau})=E_{2}(\tau)-\frac{3}{\operatorname{Im} \tau}
$$

By promoting $F_{g}(\tau)$ to the non-holomorphic modular form $F_{g}(\tau, \bar{\tau})$ in this way, the nonholomorphicity comes in only through $E_{2}$. It then follows from the holomorphic anomaly (3.1) that $(g \geq 2)$

$$
\frac{d f_{g}}{d E_{2}}=-\frac{1}{3}\left\{d_{\xi}^{2} f_{g-1}+\frac{1}{3} \frac{\partial_{\tau} \xi}{\xi} d_{\xi} f_{g-1}+\sum_{r=1}^{g-1} d_{\xi} f_{r} d_{\xi} f_{g-r}\right\}
$$

where the covariant derivative $d_{\xi}$ transforms a form of weight $k$ to a form of weight $k+2: d_{\xi}=$ $\partial_{\tau}+\frac{k}{3} \frac{\partial_{\tau} \xi}{\xi}$. In Appendix A we collect various formulae used in the subsequent computations.

The genus one free energy can be independently calculated by using Akemann's formula [30]. One can find that

$$
F_{1}(\tau)=-\log \eta(\tau)
$$

where $\eta(\tau)$ is the Dedekind eta function. This is the initial data needed to solve the holomorphic anomaly equation. In particular, at strong coupling $\lambda \sim \log ^{2} \kappa \gg 1$, this is expanded as

$$
F_{1}(\tau)=\frac{1}{6} \log \kappa-\frac{1}{2} \log (\log \kappa)+\mathcal{O}(1 / \kappa) .
$$




\subsection{The weight zero free energy}

We first focus on $A_{g}:=A_{g}^{(3 g-3)}$, s of modular weight zero. By using the formulae in Appendix $\mathrm{A}$, it is easy to show that they obey a simple recursion relation [16]

$$
\frac{A_{g}}{12}=-\frac{1}{3}\left((3 g-3) A_{g-1}+\sum_{r=2}^{g-2} A_{r} A_{g-r}\right) .
$$

They are the highest order terms in $F_{g}$ 's in the $x=1 / \log \kappa$ expansion. Indeed, using the modular transformation properties in Appendix A, one finds that

$$
F_{g}=\frac{(-1)^{g-1}}{4^{2 g-2}} \frac{A_{g}}{3 g-3} x^{3 g-3}+\mathcal{O}\left(x^{3 g-4}\right):=F_{g}^{[0]} x^{3 g-3}+\mathcal{O}\left(x^{3 g-4}\right)
$$

where $x=1 / \log \kappa$. As it will turn out, the leading order recursion (3.9) carries almost all the information needed to sum up the all genus free energy, when the worldsheet instanton corrections are ignored.

We can in fact resum the highest order all genus free energy

$$
F(t)=\sum_{g=2}^{\infty} F_{g}^{[0]} t^{2 g-2}
$$

where $t=-i g_{s} x^{3 / 2}$. For the convenience, we instead consider the generating function

$$
f(u):=\sum_{g=2}^{\infty} \tilde{A}_{g} u^{g}
$$

where $\tilde{A}_{g}=(g-1)(4 / 3)^{g-1} F_{g}^{[0]}$. Then it is easy to find that

$$
f(u)-\tilde{A}_{2} u^{2}=u^{2} f(u)^{\prime}+f(u)^{2}
$$

with $\tilde{A}_{2}=5 / 36$ which can be found from the initial genus one data. This can be solved to

$$
f(u)=-\frac{d}{d u^{-1}} \log H(u)
$$

where

$$
H(u)=e^{-\frac{1}{2 u}} \sqrt{1 / 2 u}\left(C_{1} K_{1 / 3}(1 / 2 u)+C_{2} I_{1 / 3}(1 / 2 u)\right),
$$

with $C_{1}$ and $C_{2}$ being integration constants. The highest order all genus free energy can then be computed as

$$
F(t)=-\int^{4 / 3 t^{2}} d u^{-1} f(u)=\log H\left(3 t^{2} / 4\right)
$$

Note that we absorbed the integration constant into $C_{1}$ and $C_{2}$. 
To fix the constants $C_{1}$ and $C_{2}$, we require the absence of the non-perturbative corrections of the type $e^{-2 / 3 t^{2}}$ for small $t$. Since $t \sim g_{s}$, this is of the order $e^{-1 / g_{s}^{2}}$ and would be the gravitational instanton or NS5-brane effect, as opposed to the D-brane effect, in the dual AdS string theory. We assume that the type IIA string theory on $A d S_{4} \times C P^{3}$ does not receive such corrections. With this assumption, there remain two choices; (1) $C_{2}=0$ or (2) $C_{1}=-(-1)^{5 / 6} C_{2} / \pi$. However, the latter choice is subject to a further non-perturbative ambiguity due to the Stokes phenomenon of the modified Bessel function $I_{1 / 3}(z)$. So we claim that the correct choice is $C_{2}=0$. The remaining constant $C_{1}$ only shifts the constant part of the genus one free energy and is not physically important.

Using the identity $\operatorname{Ai}(z)=\frac{1}{\pi} \sqrt{\frac{z}{3}} K_{1 / 3}\left(\frac{2}{3} z^{3 / 2}\right)$, we find

$$
F(t)=\log \left[2 \pi C_{1} e^{-\frac{2}{3 t^{2}}} t^{-\frac{1}{3}} \operatorname{Ai}\left(t^{-4 / 3}\right)\right],
$$

where $t=-i g_{s} x^{3 / 2}$. Note that the factors in front of the Airy function are precisely the minus of the highest order terms (in $x$ ) of the genus zero and one contributions. Hence the total weight zero free energy becomes simply

$$
\widehat{F}^{[0]}\left(g_{s}, x\right)=\log \left[2 \pi C_{1} \operatorname{Ai}\left(\left(-g_{s}^{2} x^{3}\right)^{-2 / 3}\right)\right] .
$$

As we will see momentarily, we have actually resummed a significant part of the all genus free energy up to the worldsheet instanton corrections.

\section{The all genus free energy}

It was observed in [11] that the all genus free energy, apart from the worldsheet instanton corrections, depends on the 't Hooft coupling only through the shifted/renormalized form

$$
\lambda_{\text {ren }}=\lambda-\frac{1}{24}-\frac{\lambda^{2}}{3 N^{2}} .
$$

This translates to the renormalization of $g_{s} x$ by

$$
g_{s} x \rightarrow g_{s} y \equiv \frac{g_{s} x}{\sqrt{1+\left(g_{s} x\right)^{2} / 6}},
$$

where $g_{s}=2 \pi i \lambda / N$ and $x^{-1}=\sqrt{2} \pi \sqrt{\lambda-1 / 24}$.

We now claim that, apart from the worldsheet instanton corrections, the all genus free energy can be obtained by replacing $x$ with the renormalized variable $y$ in the weight zero free energy (3.18). That is, the all genus free energy of the ABJM theory is given by

$$
F^{\mathrm{ABJM}}(\lambda, N)=\log \left[2 \pi C_{1} \operatorname{Ai}\left(\left(-g_{s}^{2} y^{3}\right)^{-2 / 3}\right)\right]+\mathcal{O}\left(e^{-2 \pi \sqrt{\lambda-\frac{1}{24}}}\right)
$$


where $C_{1}=\left(-g_{s}^{2}\right)^{-1 / 6} / \sqrt{2}$ to match the normalization in [11]. This is the main result (1.1).

To show it, we first note that, when the worldsheet instanton corrections, i.e., the $\mathcal{O}(1 / \kappa)$ terms, are neglected, the holomorphic anomaly equation (3.6) becomes

$$
F_{g}^{\prime}(x)=\frac{1}{4} x^{4} F_{g-1}^{\prime \prime}(x)+\frac{12 x-1}{12} x^{2} F_{g-1}^{\prime}(x)+\frac{x^{4}}{4} \sum_{r=2}^{g-2} F_{r}^{\prime}(x) F_{g-r}^{\prime}(x) .
$$

To derive this, we performed the modular transformation $S T^{-1}: \tau \mapsto \tau^{\prime}=-1 /(\tau-1)$, and kept only the terms in powers of $\log \kappa=\pi i \tau^{\prime} / 2+\mathcal{O}\left(e^{2 \pi i \tau^{\prime}}\right)$. We then used the formulae listed in Appendix A.

To proceed, we define the generating function

$$
\mathcal{F}\left(g_{s}, x\right):=\sum_{g=2}^{\infty} g_{s}^{2 g-2} F_{g}^{\prime}(x)
$$

which obeys

$$
\mathcal{F}\left(g_{s}, x\right)-g_{s}^{2} F_{2}^{\prime}(x)=\frac{1}{4} g_{s}^{2} x^{4} \partial_{x} \mathcal{F}\left(g_{s}, x\right)+\frac{12 x-1}{12} g_{s}^{2} x^{2} \mathcal{F}\left(g_{s}, x\right)+\frac{1}{4} g_{s}^{2} x^{4} \mathcal{F}\left(g_{s}, x\right)^{2},
$$

where

$$
F_{2}(x)=\frac{x}{144}-\frac{x^{2}}{24}+\frac{5 x^{3}}{48}
$$

Then the claim (4.3) is equivalent to

$$
\mathcal{F}\left(g_{s}, x\right)=\frac{\partial y}{\partial x}\left\{\frac{4}{g_{s}^{2} y^{4}} \widehat{f}\left(\frac{3}{4} g_{s}^{2} y^{3}\right)-\partial_{y}\left[\frac{2}{3 g_{s}^{2} x^{3}}+\left(\frac{1}{6 x}+\frac{1}{2} \log x\right)\right]\right\},
$$

where

$$
\widehat{f}(z):=f(z)+\left[-\frac{1}{2}+\frac{z}{6}\right] .
$$

is the generating function (3.14) plus the genus zero and one contributions, while the second term in (4.8) is the subtraction of the genus zero and one contributions. Plugging (4.8) into (4.6) yields

$$
f\left(\frac{3}{4} g_{s}^{2} y^{3}\right)-\frac{5}{36}\left(\frac{3}{4} g_{s}^{2} y^{3}\right)^{2}=\left(\frac{3}{4} g_{s}^{2} y^{3}\right)^{2} f^{\prime}\left(\frac{3}{4} g_{s}^{2} y^{3}\right)+f\left(\frac{3}{4} g_{s}^{2} y^{3}\right)^{2} .
$$

This is equivalent to (3.13). Hence we have shown that the all genus free energy (4.3) indeed satisfies the holomorphic anomaly equation (3.6) up to the worldsheet instanton corrections. 


\section{Discussions and conclusions}

We succeeded to sum up the all genus free energy of the ABJM theory, apart from the worldsheet instanton corrections. The resummation was done in two steps; (1) First, we summed up the modular weight zero part of the all genus free energy as given in (3.18) . (2) Then the remaining parts were resummed to (4.3) by renormalizing the 't Hooft coupling as was done in [11. The renormalization (1.2) agrees with the SUGRA prediction [17] in the large $N$ limit. However, there seems to be a discrepancy at the non-planar level; The matrix model suggests a further shift $-3 \lambda^{2} / 8 N^{2}$ to the string/M-theory prediction (1.3).

The quantum gravity one-loop shift $+\lambda^{2} / 24 N^{2}$ of the AdS radius in (1.3) accounts for, at the least, a part of the one-loop corrections. This comes from the shift of the M2/D2-brane charge [17]

$$
N \rightarrow N-\frac{1}{24}\left(k-\frac{1}{k}\right)=k\left(\lambda-\frac{1}{24}+\frac{\lambda^{2}}{24 N^{2}}\right),
$$

due to the higher curvature correction $C_{3} \wedge I_{8}$, where $I_{8}$ is a curvature 8-form anomaly polynomial [31]. One might wonder if the one-loop discrepancy $-3 \lambda^{2} / 8 N^{2}$ comes from other SUGRA one-loop corrections such as the $R^{4}$ term [32] 4 However, we now argue that they are absent: In fact, the one-loop $R^{4}$ term is nonzero and of the order $\sqrt{\lambda}$ which agrees with the leading part of the genus one free energy in (3.8). However, the numerical factor does not agree with the $-3 \lambda^{2} / 8 N^{2}$ discrepancy, and the $R^{4}$ term is not the only one-loop correction. The one-loop $R^{4}$ term is expected to be completed by the terms involving the 4 -form $F_{4}$. Indeed, the $11 \mathrm{~d} \mathcal{N}=1$ SUGRA on-shell superfield [33] suggests schematically the completion of the form $\left(R+F_{4}^{2}\right)^{4}$, but this vanishes on $A d S_{4} \times S^{7} / Z_{k}$ [34, 35]. Furthermore, the one-loop vacuum energy vanishes in $4 \mathrm{~d} \mathcal{N} \geq 5$ SUGRA on the global $A d S_{4}$ whose boundary is $\mathbb{R} \times S^{2}$ [36]. Although we work in the Euclidean $A d S_{4}$ with the boundary $S^{3}$, the localizations of the ABJM theory on $S^{3}$ and $\mathbb{R} \times S^{2}$ turn out to be the same [37]. So we expect the SUGRA result on the global $A d S_{4}$ applies to our case, and it seems likely that the one-loop corrections, except for the charge shift (5.1), are absent.

This leaves us the $-3 \lambda^{2} / 8 N^{2}$ discrepancy and suggests us to look for the resolution on the matrix model side. Recall that ABJM proposed two $\mathcal{N}=6$ superconformal ChernSimons-Matter theories 88; One has the gauge group $U(N) \times U(N)$ and the other has $S U(N) \times S U(N)$. In the large $N$ limit, their difference may not matter. But at finite $N$ they will differ from each other in the $1 / N$ corrections. In fact, in the $A d S_{5} \times S^{5}$ case, the correct gauge group is $S U(N)$ rather than $U(N)$ [38]. Although the status of this subtlety is unclear in the $A d S_{4}$ case, it may be worthwhile to investigate $S U(N) \times S U(N)$ ABJM matrix model to see if the $-3 \lambda^{2} / 8 N^{2}$ discrepancy can be resolved. We hope to address this issue further in the near future.

\footnotetext{
${ }^{4}$ The $\lambda^{2} / N^{2}$ shift appears of the order $\sqrt{\lambda}$ in the genus one free energy.
} 
Finally, it is worth emphasizing the remarkable simplicity of our result; The partition function of the ABJM theory, when the worldsheet instantons are neglected, is simply the Airy function. This might suggest a possible connection of the ABJM theory to the Kontsevich matrix model [39] upon the inclusion of the worldsheet instanton corrections. Meanwhile, in a somewhat different context of M-theory flux compactification, it was argued that the norm square of the "wave-function of the universe" or the $5 \mathrm{~d}$ black hole entropy was given by the Airy function [40] 5 It would be interesting to study the relations to these works in the future.

\section{Acknowledgment}

We would like to thank Oren Bergman, Nadav Drukker, Kazuo Hosomichi, Hiroaki Kanno, Masahide Manabe, Kazuhiro Sakai, and Masaki Shigemori for useful discussions and conversations. This work was partially supported by the Grant-in-Aid for Nagoya University Global COE Program (G07), by that for Young Scientists (B) [\# 21740179] (H.F.) and by [\# 21740176] (S.M.) from the Ministry of Education, Culture, Sports, Science and Technology of Japan.

\section{A Formulae for holomorphic anomaly equation}

In this appendix, we gather various useful formulae related to the holomorphic anomaly equation:

$$
\begin{aligned}
& b \equiv \vartheta_{2}(\tau)^{4}, \quad c \equiv \vartheta_{3}(\tau)^{4}, \quad d \equiv \vartheta_{4}(\tau)^{4}, \\
& \frac{\partial_{\tau} \xi}{\xi}=\frac{b-E_{2}}{4}, \quad d_{\xi} f_{1}=-\frac{E_{2}}{24}, \quad d_{\xi} b=\frac{1}{3} b(b+d), \quad d_{\xi} d=-\frac{1}{6} d(b+d), \\
& d_{\xi} E_{2}=\frac{2 b E_{2}-E_{2}^{2}-E_{4}}{12}, \quad d_{\xi} E_{4}=\frac{b E_{4}-E_{6}}{3}, \quad d_{\xi} E_{6}=\frac{b E_{6}-E_{4}^{2}}{2} .
\end{aligned}
$$

The following formulae are useful to derive the weight zero recursion (3.9)

$$
\begin{aligned}
f_{g} & =\frac{A_{g}^{(3 g-3)}}{3 g-3}\left(\frac{E_{2}}{12}\right)^{3 g-3}+\cdots, \\
d_{\xi} f_{g} & =-A_{g}^{(3 g-3)}\left(\frac{E_{2}}{12}\right)^{3 g-2}+\cdots, \\
d_{\xi}^{2} f_{g} & =(3 g-2) A_{g}^{(3 g-3)}\left(\frac{E_{2}}{12}\right)^{3 g-1}+\cdots .
\end{aligned}
$$

To study the perturbative terms as in (4.4), we shall use the modular transformations $S T^{-1}$ : $\tau \mapsto \tau^{\prime}=-1 /(\tau-1)$, since (3.2) implies that $\tau$ is given by $\tau=1+\pi i /(2 \log \kappa)$, neglecting

\footnotetext{
${ }^{5}$ The authors are grateful to Hirosi Ooguri for pointing this out to us. See also [41].
} 
the $\mathcal{O}(1 / \kappa)$ instanton terms,

$$
\begin{aligned}
b(\tau) & =-\left(-i \tau^{\prime}\right)^{2} d\left(\tau^{\prime}\right)=\left(\tau^{\prime}\right)^{2}[1+\mathcal{O}(1 / \kappa)], \\
d(\tau) & =\left(-i \tau^{\prime}\right)^{2} c\left(\tau^{\prime}\right)=\left(\tau^{\prime}\right)^{2}[-1+\mathcal{O}(1 / \kappa)], \\
E_{2}(\tau) & =\left(\tau^{\prime}\right)^{2}\left[E_{2}\left(\tau^{\prime}\right)-6 i /\left(\pi \tau^{\prime}\right)\right]=\left(\tau^{\prime}\right)^{2}[(1-3 x)+\mathcal{O}(1 / \kappa)], \\
\xi^{2}(\tau) & =4 /\left(b(\tau) d(\tau)^{2}\right)=\left(\tau^{\prime}\right)^{-6}[4+\mathcal{O}(1 / \kappa)],
\end{aligned}
$$

with $x=1 / \log \kappa$. Note that since the holomorphic anomaly equation is a modular covariant equation, the modular scale factors $\tau^{\prime}$ are destined to be cancelled out in the end. We

shall drop them in our following analysis. Then, we obtain the following formulae in the approximation neglecting the $\mathcal{O}(1 / \kappa)$ terms:

$$
d_{\xi} E_{2}=-\frac{3}{4} x^{2}, \quad d_{\xi}^{2} E_{2}=-\frac{3}{8} x^{3}, \quad \frac{1}{3} \frac{\partial_{\tau} \xi}{\xi}=\frac{x}{4}, \quad \frac{d E_{2}}{d x}=-3, \quad d_{\xi} b=d_{\xi} d=0 .
$$

From these we find

$$
\begin{aligned}
& \frac{d f_{g}}{d E_{2}}=-\frac{1}{3} f_{g}^{\prime}(x) \\
& d_{\xi} f_{g}=\left(d_{\xi} E_{2}\right) \frac{d f_{g}}{d E_{2}}=\frac{x^{2}}{4} f_{g}^{\prime}(x) \\
& d_{\xi}^{2} f_{g}=\left(d_{\xi}^{2} E_{2}\right) \frac{d f_{g}}{d E_{2}}+\left(d_{\xi} E_{2}\right)^{2} \frac{d^{2} f_{g}}{d E_{2}^{2}}=\frac{x^{3}}{8} f_{g}^{\prime}(x)+\frac{x^{4}}{16} f_{g}^{\prime \prime}(x) .
\end{aligned}
$$

\section{B A derivation of weight zero free energy}

In this appendix we solve the differential equation (3.13): Introducing the new variable $v=1 /(2 u)$ and the new function

$$
f=-\frac{1}{2} \frac{d}{d v} \log H
$$

this becomes

$$
\left[\frac{d^{2}}{d v^{2}}+2 \frac{d}{d v}+\frac{\tilde{A}_{2}}{v^{2}}\right] H=0 .
$$

In terms of $G(v)=e^{v} v^{-\frac{1}{2}} H(v)$, one obtains $(\alpha=1 / 3)$

$$
\left[v^{2} \frac{d}{d v}+v \frac{d}{d v}-\left(v^{2}+\alpha^{2}\right)\right] G=0,
$$

which is nothing but the modified Bessel's differential equation. The solution is given by the modified Bessel's functions: $G(v)=C_{1} K_{1 / 3}(v)+C_{2} I_{1 / 3}(v)$. 


\section{References}

[1] J. M. Maldacena, "The large N limit of superconformal field theories and supergravity," Adv. Theor. Math. Phys. 2, 231 (1998) [Int. J. Theor. Phys. 38, 1113 (1999)] arXiv:hep-th/9711200;

[2] N. Beisert, C. Ahn, L. F. Alday, Z. Bajnok, J. M. Drummond, L. Freyhult, N. Gromov, R. A. Janik, V. Kazakov, T. Klose, G. P. Korchemsky, C. Kristjansen, M. Magro, T. McLoughlin, J. A. Minahan, R. I. Nepomechie, A. Rej, R. Roiban, S. Schafer-Nameki, C. Sieg, M. Staudacher, A. Torrielli, A. A. Tseytlin, P. Vieira, D. Volin, K. Zoubos, "Review of AdS/CFT Integrability: An Overview," arXiv:1012.3982 [hep-th]].

[3] N. Beisert, C. Kristjansen and M. Staudacher, "The Dilatation operator of conformal N=4 superYang-Mills theory," Nucl. Phys. B 664, 131 (2003) arXiv:hep-th/0303060; C. Kristjansen, M. Orselli and K. Zoubos, "Non-planar ABJM Theory and Integrability," JHEP 0903, 037 (2009) arXiv:0811.2150 [hep-th]]; P. Caputa, C. Kristjansen and K. Zoubos, "Non-planar ABJ Theory and Parity," Phys. Lett. B 677, 197 (2009) arXiv:0903.3354 [hep-th]].

[4] V. Pestun, "Localization of gauge theory on a four-sphere and supersymmetric Wilson loops," arXiv:0712.2824 [hep-th].

[5] J. K. Erickson, G. W. Semenoff and K. Zarembo, "Wilson loops in N=4 supersymmetric Yang-Mills theory," Nucl. Phys. B 582, 155 (2000) arXiv:hep-th/0003055].

[6] N. Drukker and D. J. Gross, "An Exact prediction of N=4 SYM theory for string theory," J. Math. Phys. 42, 2896 (2001) arXiv:hep-th/0010274.

[7] A. Kapustin, B. Willett and I. Yaakov, "Exact Results for Wilson Loops in Superconformal Chern-Simons Theories with Matter," JHEP 1003, 089 (2010) arXiv:0909.4559 [hep-th]].

[8] O. Aharony, O. Bergman, D. L. Jafferis and J. Maldacena, "N=6 superconformal ChernSimons-matter theories, M2-branes and their gravity duals," arXiv:0806.1218 [hep-th].

[9] N. Drukker, M. Marino and P. Putrov, "From weak to strong coupling in ABJM theory," arXiv:1007.3837 [hep-th].

[10] M. Marino, "Lectures on localization and matrix models in supersymmetric ChernSimons-matter theories," arXiv:1104.0783 [hep-th]].

[11] N. Drukker, M. Marino and P. Putrov, "Nonperturbative aspects of ABJM theory," arXiv:1103.4844 [hep-th]. 
[12] C. P. Herzog, I. R. Klebanov, S. S. Pufu and T. Tesileanu, "Multi-Matrix Models and Tri-Sasaki Einstein Spaces," Phys. Rev. D 83, 046001 (2011) arXiv:1011.5487 [hep-th]]; D. Martelli and J. Sparks, "The large N limit of quiver matrix models and Sasaki-Einstein manifolds," arXiv:1102.5289 [hep-th]; S. Cheon, H. Kim and N. Kim, "Calculating the partition function of $\mathrm{N}=2$ Gauge theories on $S^{3}$ and AdS/CFT correspondence," JHEP 1105, 134 (2011) [arXiv:1102.5565 [hep-th]].

[13] D. L. Jafferis, "The Exact Superconformal R-Symmetry Extremizes Z," arXiv:1012.3210 [hep-th]; D. L. Jafferis, I. R. Klebanov, S. S. Pufu and B. R. Safdi, "Towards the F-Theorem: N=2 Field Theories on the Three-Sphere," arXiv:1103.1181 [hep-th]; A. Amariti and M. Siani, "Z-extremization and F-theorem in Chern-Simons matter theories," arXiv:1105.0933 [hep-th]; A. Amariti and M. Siani, "F-maximization along the RG flows: A Proposal," arXiv:1105.3979 [hep-th].

[14] Y. Imamura and S. Yokoyama, "Index for three dimensional superconformal field theories with general R-charge assignments," JHEP 1104, 007 (2011) arXiv:1101.0557 [hepth]]; Y. Imamura, D. Yokoyama and S. Yokoyama, "Superconformal index for large N quiver Chern-Simons theories," arXiv:1102.0621 [hep-th]; C. Krattenthaler, V. P. Spiridonov and G. S. Vartanov, "Superconformal indices of three-dimensional theories related by mirror symmetry," JHEP 1106, 008 (2011) arXiv:1103.4075 [hep-th]].

[15] A. Kapustin, B. Willett and I. Yaakov, "Nonperturbative Tests of Three-Dimensional Dualities," JHEP 1010, 013 (2010) arXiv:1003.5694 [hep-th]]; A. Kapustin, B. Willett and I. Yaakov, "Tests of Seiberg-like Duality in Three Dimensions," arXiv:1012.4021 [hep-th].

[16] M. x. Huang and A. Klemm, "Holomorphicity and Modularity in Seiberg-Witten Theories with Matter," JHEP 1007, 083 (2010) [arXiv:0902.1325 [hep-th]].

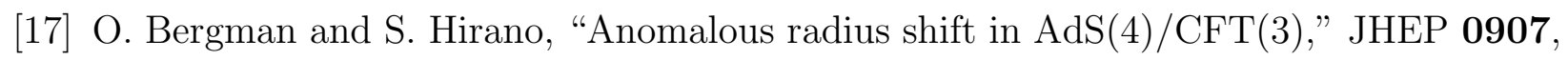
016 (2009) arXiv:0902.1743 [hep-th]].

[18] O. Aharony, O. Bergman and D. L. Jafferis, "Fractional M2-branes," arXiv:0807.4924 [hep-th].

[19] O. Aharony, A. Hashimoto, S. Hirano and P. Ouyang, "D-brane Charges in Gravitational Duals of $2+1$ Dimensional Gauge Theories and Duality Cascades," JHEP 1001, 072 (2010) [arXiv:0906.2390 [hep-th]].

[20] N. Hama, K. Hosomichi and S. Lee, "Notes on SUSY Gauge Theories on Three-Sphere," JHEP 1103, 127 (2011) arXiv:1012.3512 [hep-th]].

[21] N. Halmagyi and V. Yasnov, "The Spectral curve of the lens space matrix model," JHEP 0911, 104 (2009) arXiv:hep-th/0311117. 
[22] M. Marino and P. Putrov, "Exact Results in ABJM Theory from Topological Strings," JHEP 1006, 011 (2010) [arXiv:0912.3074 [hep-th]].

[23] M. Marino, "Chern-Simons theory, matrix integrals, and perturbative three manifold invariants," Commun. Math. Phys. 253, 25 (2004) arXiv:hep-th/0207096].

[24] M. Aganagic, A. Klemm, M. Marino and C. Vafa, "Matrix model as a mirror of ChernSimons theory," JHEP 0402, 010 (2004) arXiv:hep-th/0211098.

[25] M. Bershadsky, S. Cecotti, H. Ooguri and C. Vafa, "Kodaira-Spencer theory of gravity and exact results for quantum string amplitudes," Commun. Math. Phys. 165, 311 (1994) arXiv:hep-th/9309140.

[26] R. Dijkgraaf and C. Vafa, "Matrix models, topological strings, and supersymmetric gauge theories," Nucl. Phys. B 644, 3 (2002) arXiv:hep-th/0206255].

[27] B. Eynard, M. Marino and N. Orantin, "Holomorphic anomaly and matrix models," JHEP 0706, 058 (2007) arXiv:hep-th/0702110.

[28] B. Eynard and N. Orantin, "Invariants of algebraic curves and topological expansion," arXiv:math-ph/0702045.

[29] J. Ambjorn, L. Chekhov, C. F. Kristjansen and Yu. Makeenko, "Matrix model calculations beyond the spherical limit," Nucl. Phys. B 404, 127 (1993) [Erratum-ibid. B 449, 681 (1995)] arXiv:hep-th/9302014.

[30] G. Akemann, "Higher genus correlators for the Hermitian matrix model with multiple cuts," Nucl. Phys. B 482, 403 (1996) arXiv:hep-th/9606004.

[31] M. J. Duff, J. T. Liu and R. Minasian, "Eleven-dimensional origin of string / string duality: A one-loop test," Nucl. Phys. B 452, 261 (1995) arXiv:hep-th/9506126.

[32] M. B. Green and P. Vanhove, "D instantons, strings and M theory," Phys. Lett. B 408, 122 (1997) arXiv:hep-th/9704145; M. B. Green, M. Gutperle and P. Vanhove, "One loop in eleven-dimensions," Phys. Lett. B 409, 177 (1997) arXiv:hep-th/9706175.

[33] E. Cremmer and S. Ferrara, "Formulation of Eleven-Dimensional Supergravity in Superspace," Phys. Lett. B 91, 61 (1980); L. Brink and P. S. Howe, "Eleven-Dimensional Supergravity on the Mass-Shell in Superspace," Phys. Lett. B 91, 384 (1980).

[34] R. Kallosh and A. Rajaraman, "Vacua of M-theory and string theory," Phys. Rev. D 58, 125003 (1998) arXiv:hep-th/9805041.

[35] A. A. Tseytlin, " $\mathrm{R}^{4}$ terms in 11 dimensions and conformal anomaly of $(2,0)$ theory," Nucl. Phys. B 584, 233 (2000) arXiv:hep-th/0005072. 
[36] B. Allen and S. Davis, "Vacuum Energy in Gauged Extended Supergravity," Phys. Lett. B 124, 353 (1983).

[37] N. Hama, K. Hosomichi and S. Lee, "SUSY Gauge Theories on Squashed ThreeSpheres," JHEP 1105, 014 (2011) [arXiv:1102.4716 [hep-th]].

[38] E. Witten, "Anti-de Sitter space and holography," Adv. Theor. Math. Phys. 2, 253 (1998) arXiv:hep-th/9802150]; O. Aharony and E. Witten, "Anti-de Sitter space and the center of the gauge group," JHEP 9811, 018 (1998) arXiv:hep-th/9807205.

[39] M. Kontsevich, "Intersection theory on the moduli space of curves and the matrix Airy function," Commun. Math. Phys. 147, 1 (1992).

[40] H. Ooguri, C. Vafa and E. P. Verlinde, "Hartle-Hawking wave-function for flux compactifications," Lett. Math. Phys. 74, 311 (2005) arXiv:hep-th/0502211.

[41] M. Marino, "Exact Results and Stringy Effects in ABJM Theory," talk at the conference "Strings 2011," Uppsala, June 2011. 